\title{
The Impact of an Intervention Taught by Trained Teachers on Childhood Fruit and Vegetable Intake: A Randomized Trial
}

\author{
Rafaela Rosário, ${ }^{1,2}$ Ana Araújo, ${ }^{2}$ Bruno Oliveira, ${ }^{3}$ Patrícia Padrão, ${ }^{3}$ Oscar Lopes, ${ }^{4}$ \\ Vítor Teixeira, ${ }^{3}$ André Moreira, ${ }^{5}$ Renata Barros, ${ }^{3}$ Beatriz Pereira, ${ }^{2}$ and Pedro Moreira ${ }^{3,6}$ \\ ${ }^{1}$ School of Nursing, University of Minho, Largo do Paço, Braga, Portugal \\ ${ }^{2}$ Research Centre on Child Studies, School of Education, University of Minho, Campus de Gualtar, Braga, Portugal \\ ${ }^{3}$ Faculty of Nutrition and Food Sciences, University of Porto, Rua Dr. Roberto Frias, Porto, Portugal \\ ${ }^{4}$ Tempo Livre, Sports' Medical Center, Alameda dos Desportos Candoso S. Tiago, Guimarães, Portugal \\ ${ }^{5}$ Faculty of Medicine, University of Porto, Al. Prof. Hernâni Monteiro, Porto, Portugal \\ ${ }^{6}$ Research Centre in Physical Activity, Health, and Leisure, University of Porto, Rua Dr. Plácido Costa, Porto, Portugal
}

Correspondence should be addressed to Rafaela Rosário, rrosario@ese.uminho.pt

Received 2 November 2011; Accepted 12 December 2011

Academic Editor: Jack A. Yanovski

Copyright ( 2012 Rafaela Rosário et al. This is an open access article distributed under the Creative Commons Attribution License, which permits unrestricted use, distribution, and reproduction in any medium, provided the original work is properly cited.

\begin{abstract}
Our study aimed to assess the impact of a six-months nutrition program, taught by trained teachers, on fruit and vegetable consumption among children in grades 1 to 4 . Four hundred and sixty-four children ( 239 female), 6 to 12 years old, from seven elementary schools were assigned to this randomized trial. Teachers were trained by researchers over six months, according to the following topics: nutrition, healthy eating, and strategies to increase physical activity. After each session, teachers were encouraged to develop activities in the classroom on the topics learned. Children's sociodemographic, anthropometric, dietary, and physical activity data were assessed at baseline and at the end of the intervention. The effect sizes ranged between small (Cohen's $d=0.12$ on "other vegetables") to medium ( 0.56 on "fruit and vegetable"), and intervened children reported a significantly higher consumption of vegetables and fruit. Interventions involving trained teachers offer promise to increase consumption of fruit and vegetable in children.
\end{abstract}

\section{Introduction}

Fruit and vegetable consumption has been shown to be associated with a reduced risk of obesity [1], cancer [2], asthma [3], cardiovascular disease, and higher life expectancy $[4,5]$. Current dietary recommendations of fruit and vegetables promote a consumption of at least five servings of fruit and vegetables a day, or a minimum intake of $400 \mathrm{~g}$ of fruit and vegetables $[6,7]$. However, dietary consumption surveys show that most European children do not meet these guidelines [8-10]. There is a need to develop and implement effective programs able to improve children eating habits.

The best practice to promote fruit and vegetable consumption is far from complete. Evidence suggests that the childhood period represents an important opportunity to achieve the recommended intake of fruit and vegetable, because eating patterns are still being developed [11] and might track into adulthood [12-14]. In addition, schools are well recognized as a crucial setting for interventions to promote healthful eating habits [15-17]; however until now there is scant evidence of the role of teachers in the accomplishment of programs [16, 17]. Theoretically, teachers are not able to devote as much time and energy for provision of interventions, like dedicated interventionists do, because they have classroom responsibilities that take precedence [16]. Moreover, there are few, if any, examples of studies that included the program in the progression of teaching career, and the role of educational interventions in improving dietary habits in youth in Europe is unclear. Few previous studies examined the effects on the consumption of 
fruit and vegetable in children [18-25], and only two of them were considered effective $[23,24]$.

The main goal of the present research was to assess the impact of a six-month nutrition program, taught by trained teachers on nutrition, on fruit and vegetable consumption among children in grades 1 to 4 .

\section{Methods}

2.1. Participants. During 2007/2008 seven out of eighty public elementary schools were randomly selected and invited to participate in this study. The number of schools involved was according to constraints of personnel for the assessment and implementation of the program. Schools were the unit of randomization, and three were assigned into intervention, and four into control group (Figure 1). Data was collected before and immediately after the program, during the year 2009 (after intervention). Prior to data collection, parents provided informed consent, in accord with the ethical standards laid down in the Declaration of Helsinki, and children gave oral assent. The study was approved by the schools and by the Portuguese Data Protection Authority (CNPD_Comissão Nacional de Protecção de Dados). In addition the protocol of this study was registered in the clinical trials registry clinicaltrials.gov, NCT01397123.

The flow of the subjects during the study is presented in Figure 1. From the 574 children who were invited to participate, 464 (239 female), age ranged 6 to 12 years old, agreed and returned $(80.8 \%)$ the written consent forms filled by their parents. From these, $233(50.2 \%)$ were allocated to the intervention group, and $231(49.8 \%)$ to the control group. Follow-up assessment was available for $63.4 \%$ of the children, $143(61.9 \%)$ in the control and $151(64.8 \%)$ in the intervention groups. Attrition rates did not differ between intervention and control group (35.2\% and 38.1\%, resp.). Major reasons for nonparticipation were school transfer (94.1\%), parental refusal $(4.1 \%)$, and absence from school (1.8\%). Children and outcomes assessors were blinded to group assignment. A total of 257 parents of the children involved in the study provided data at baseline and 203 (79.0\%) after intervention.

2.2. Overview of the Program. Teachers from intervention schools were invited and agreed to participate in the program conducted between October 2008 and March 2009; fifteen teachers were involved. This program was based on the Health Promotion Model [26] and the social cognitive theory [27] and aimed to promote healthier active lifestyles by encouraging children to be more active and ensure better food selection.

The Health Promotion Model argues that actions and the health promotion behavior are a corollary of personal characteristics, prior experiences, perceived benefits, and barriers for action as well as perceived self-efficacy [26]. This program was aware of these influences on children's behavior and focused on a positive vision of health. Likewise, the social cognitive theory enhanced cognitive and behavioral skills by enabling children to make changes in their own behavior and employing new choices effectively [27].
The research team proposed, in January 2008, the accreditation of the training sessions developed with the teachers to the Minister of Education, Scientific-Pedagogic Council for In-Service Training (Conselho Científico Pedagógico da Formação Contínua, Ministério da Educação). This proposal was approved in September 2008 in the form of "training workshop" with 72-hour duration, distributed by active learning strategies (36 hours with the researchers) and delivery (intervention) of the learnt contents to the children (36 hours). Thus, the program was implemented over two terms: teachers' training delivered by researchers and the intervention delivered by trained teachers to children.

Teachers of the intervention group had 12 sessions of three hours each with the researchers throughout six months, which included the following topics: nutrition and healthy eating for children and families (sessions 1 to 4 ); the importance of water (session 5); strategies to encourage fruit and vegetable consumption and to reduce low-nutrition, energy-dense foods (sessions 6 to 8 ); to increase physical activity and to reduce screen time exposure (sessions 9 and 10); healthy cooking activities (sessions 11 and 12). After each session, teachers delivered the learnt contents and developed creative and engaging classroom activities about the addressed topic. All the questions that arose during the implementation of classroom activities were addressed and resolved shortly with the researchers. Teachers were allowed to develop and refine the activities and the learning strategies that were proposed by researchers. At the end of this period, teachers delivered a critical report of activities focused on the intervention.

The implementation of the program occurred as planned. All the children of the intervention schools had contact with trained teachers who taught the components of the program as prescribed. Teachers reported they were enthusiastic about the training, and had a total attendance in the sessions with the researchers. In addition, researchers were always available to answer any question.

2.3. Assessments. In each school, previously trained persons performed anthropometric evaluation, using standardized procedures [28]. Anthropometric measurements were performed in children with light indoor clothing and barefooted. Weight was measured in an electronic scale, with an error of $\pm 100 \mathrm{~g}$ (Seca, Model 703, Germany), and height was measured using a stadiometer, with the head in the Frankfort plane. BMI was computed as mass, $(\mathrm{Kg}) /$ height $^{2}(\mathrm{~m})$. The prevalence of underweight, normal weight, overweight, and obesity was calculated according to the International Obesity Task Force (IOTF) criteria, making a correspondence between the traditional adult cutoff and specific values for children according to gender and age [29]. A z-score (the number of standard deviations ( $\mathrm{sd}$ ) from the reference population) was calculated for each child using the LMS method and the calculation was determined using the LMS growth add-in for Excel [30].

Dietary intake was gathered by a 24-hour dietary recall obtained by nutritionists and/or trained interviewers one before and one after the intervention. Training of interviewers included practice using photos and food models to 


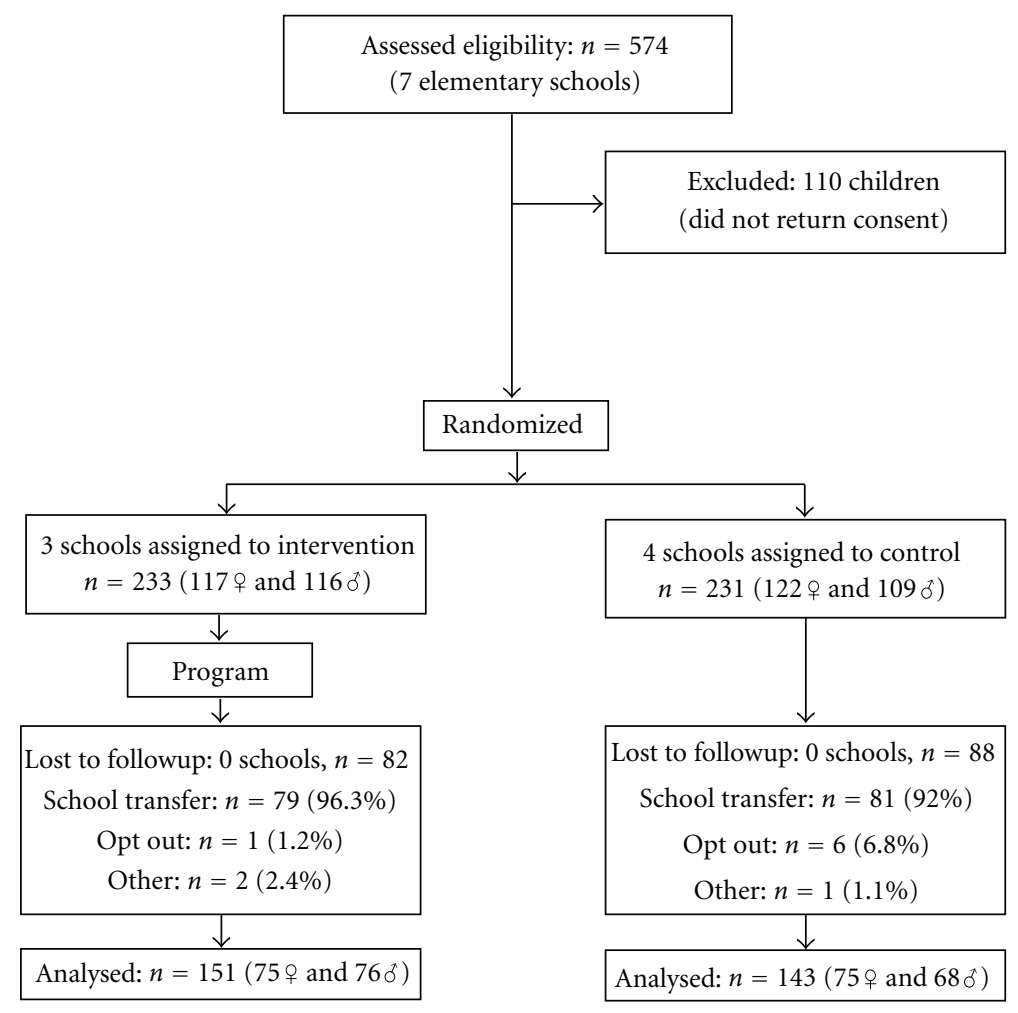

FIGURE 1: Flow of participants through each stage of the program.

quantify portion sizes, and experience in probing information from children without suggesting responses as well as the type of food consumed in detail of fat content, brand name, constituents of mixed dishes, and so on. The $24 \mathrm{~h}$ dietary recall is the most commonly used dietary assessment method because it is easy to administer, can be performed in large-scale studies [31, 32], and can be used to assess adequacy of energy and macronutrient intakes. Children did not have prior notification of when the recalls would occur in order to prevent potentially biasing reports and the weekend days were avoided. During the $24 \mathrm{~h}$ dietary recall, each child was asked to recall all food and beverages consumed during the past $24 \mathrm{~h}$. Daily routines were used as prompts (waking up, going to bed, time between classes, and before or after school) to enhance recall. Portion sizes of foods and beverages consumed were also estimated using food models, photos, and other props (cups, glasses, food wrappers, or containers) as an aid in determining serving sizes. Energy and nutritional intake were estimated using an adapted Portuguese version of the nutritional analysis software Food Processor Plus (ESHA Research Inc., Salem, OR, USA).

The 24-hour dietary recall interviews captured the time, type, local and the foods and beverages at each eating occasion, and the source from which item was obtained. The results from the 24-hour dietary recall regarding vegetables were merged into four subgroups, that is, legumes (bayo beans, black beans, blackeyed peas, lentils, navy beans, etc.), leafy vegetables (kale, lettuce, parsley, poke greens, spinach, etc.), orange vegetables (carrots, pumpkin, winter squash, yams, etc.), and vegetable soup. To estimate vegetables consumption, total vegetables (legumes, leafy vegetables, and orange vegetables) and other vegetables were also considered (legumes and orange vegetables); vegetable soup was not considered in total vegetable intake, and was analysed as a separate variable. Regarding fruit, the results were merged into three subgroups, that is, whole fruit, fruit juice, and total fruit, which include whole fruit and fruit juice. In order to estimate the magnitude of fruit and vegetables consumption changes during the study, new variables were computed from the difference between the postintervention and baseline for total vegetables, leafy vegetables, other vegetables, vegetable soup, total fruit, whole fruit, fruit juice, and fruit and vegetables. Thus, a positive difference score represents change in the expected direction for all the outcomes.

To evaluate the mean population bias in reported energy intake, at baseline and after intervention, the ratio Energy intake (EI) : basal metabolic rate (BMR) was computed for each subject, according to gender and age-specific equation [33] adopted by the FAO/WHO/UNU report [34]. BMR was determined through the Schofield equations and the subjects with $\mathrm{EI}: \mathrm{BMR} \leq 0.89$ were classified as low energy reporters (LER) and excluded from analysis [35].

In order to assess the level of physical activity of children, parents were asked five questions with four answer choices (4-point scale): (a) Outside the school does your child take part in organized sport? (b) Outside school does your child take part in nonorganized sport? (c) Outside school, how many times a week does your child take part in sport or 
physical activity for at least 20 minutes? (d) Outside school hours, how many hours a week does your child usually take part in physical activity so much that he gets out of breath or sweat? (e) Does your child take part in competitive sport? [36]. The total sum of the points was computed reaching a maximum of 20 points. A Physical Activity Index was obtained, which divided the sample into four activity classes levels: sedentary group (5); low activity group (6-10); moderately active group (11-15); vigorously active group (16-20), on the basis of their reported physical activity [36].

Social, demographics and family characteristics were assessed by questionnaire. The survey sent to parents contained questions about gender and age of the children, education of the parents (recorded in five categories: $0,1-$ $4,5-9,10-12$, and more than 12 years of formal education). This information was further grouped for analysis into three categories: up to 9 years, $10-12$ years, and more than 12 years of education.

2.4. Statistical Analyses. Mean and standard deviations (sd) were used to describe continuous variables. Student's $t$-tests, Mann-Whitney U, Kruskall-Wallis, and Chi-square tests were used to compare several variables grouped by intervention and control school groups and sex. These tests were also conducted to assure comparability of fruit and vegetables consumption between groups at baseline. A 0.05 level of significance was considered.

Effect sizes were evaluated using Cohen's $d$ as a measure that reflects the magnitude of the difference between groups in sd units. To account for the different conditions at baseline, and to rule out the possibility that these differences, even if nonsignificant, artificially amplify or attenuate effect sizes estimates, we used the adjusted means and the adjusted sd, controlled for baseline conditions. Subtracting the adjusted means for control and intervention groups and dividing by the adjusted sd for the measure compute Cohen's $d$. We use the standard criteria for effect sizes of small $(d=0.20)$, medium $(d=0.50)$, and large effects $(d=0.80)$ [37].

The effect of the program was evaluated based on changes in fruit and vegetable consumption between baseline and after intervention, comparing intervention to control schools. The tests examining these differences were developed using Generalized Linear Models and took into account the nested nature of the data (children were nested within schools). Hence, the difference scores from total vegetables, leafy vegetables, other vegetables, total fruit, whole fruit, fruit juice, fruit and vegetables, and vegetable soup were used as dependent variable, and the adjustment was made for gender, age, parents' education, baseline levels of energy intake, BMI, and baseline measures of the dependent variable in order to maximize precision.

The data analysis was performed using SPSS, version 18.0 (SPSS Inc. Chicago, IL).

\section{Results}

Table 1 shows the characteristics of the participants, before and after the intervention. At baseline, subjects included
239 girls and 225 boys with a mean (SD) age of 8.3 (1.2) years. There was no interaction by gender; therefore, data are shown from boys and girls combined.

The average BMI was $17.9(3.4) \mathrm{kg} / \mathrm{m}^{2}$ ranging from 11.9 to $26.9 \mathrm{~kg} / \mathrm{m}^{2}$ and BMI $z$-score was 0.8 (1.1). Overall, $23.3 \%$ of the children were classified as overweight, $55(11,9 \%)$ boys and $53(11,4 \%)$ girls, and $9.5 \%$ as obese, $22(4,7 \%)$ boys and $22(4,7 \%)$ girls. The large majority $(\approx 65 \%)$ of the children was classified as sedentary or having low activity. Mean energy intake was not significantly different between intervention and control groups (resp., 2091 (684) kcal versus 2024 (582) kcal, $P=0.257$ ).

There were significant differences between groups with regard to mother $(P=0.021)$ and father $(P=0.030)$ education levels, which were higher in the intervention group. To account for these differences at baseline, these variables were controlled for in subsequent analyses.

Sixteen (3.4\%) children were found as LER at baseline and $5(1.7 \%)$ after the intervention, and no significant differences between control and intervention groups were found.

At baseline, there were no significant differences on the consumption of fruit and vegetable between control and intervention group.

We analyzed the effect of the program on food consumption, hypothesizing that children in the intervention group would have greater improvements in fruit and vegetable intake.

In Generalized Linear Models, when adjusted for gender, age, baseline total energy intake, parents' education, baseline BMI, and baseline values of the dependent variable, there was a significant higher increase in total vegetables and green leafy vegetables intake in intervention than in the control group. In addition, a significant increase was observed in the intake of total fruit, and fruit and vegetable in the intervention group while the control group had a significant decrease in their consumption ( Table 2).

The adjusted effect sizes ranged from small to medium (Cohen's $d$ ranged from 0.12 , in other vegetables, to 0.56 , in fruit and vegetable together). Despite a lack of effect sizes, the differences are in the expected direction.

We examined if the effect of the program was modified by child's weight status, hypothesizing that obese children (overweight and obese) could benefit most from the program. These analyses examined the effect of program, weight status, and the program by weight status interaction on the outcome measures, controlling for baseline values. Intervened obese children consumed significantly higher amounts of total vegetables (adjusted mean (standard error), 81.9 (16.4)), than controlled obese children (11.6 (22.1), $P=0.046$ ) (data not shown). Likewise, intervened obese children reported significantly greater consumption of green leafy vegetable $(41.5(8.8))$ versus -4.6 (11.9) in control schools, $P=0.016$. This trend was the same on fruit consumption because intervened obese children reported a significantly greater consumption of total fruit (115.3 (46.5)) compared to control $(-168.2$ (65.1)), $P=0.030$; whole fruit (52.7 (38.7) compared to control -161.4 (53.1), $P=0.011)$, as well as on fruit and 
TABLE 1: Anthropometric and sociodemographic characteristics at baseline and after intervention.

\begin{tabular}{|c|c|c|c|c|c|c|}
\hline & \multicolumn{2}{|c|}{ Baseline } & \multicolumn{4}{|c|}{ After intervention } \\
\hline & Intervention $(n=231)$ & Control $(n=233)$ & $P$ & Intervention $(n=151)$ & Control $(n=143)$ & $P$ \\
\hline Age $(\text { years })^{1}$ & $8.3(1.2)$ & $8.2(1.2)$ & 0.846 & $9.2(0.9)$ & $9.1(1.0)$ & 0.494 \\
\hline Boys, $n(\%)^{2}$ & $116(49.8)$ & $109(47.2)$ & & $76(50.3)$ & $68(47.6)$ & \\
\hline Girls, $n(\%)^{2}$ & $117(50.2)$ & $122(52.8)$ & 0.575 & $75(49.7)$ & $75(52.4)$ & 0.634 \\
\hline \multicolumn{7}{|l|}{ Mother's education, $n(\%)^{2}$} \\
\hline Up to 9 years & $116(58.6)$ & $128(69.9)$ & & $77(59.2)$ & $81(69.8)$ & \\
\hline $10-12$ years & $52(26.3)$ & $36(19.7)$ & & $32(24.6)$ & $26(22.4)$ & \\
\hline$>12$ years & $30(15.2)$ & $19(10.4)$ & 0.021 & $21(16.2)$ & $9(3.7)$ & 0.050 \\
\hline \multicolumn{7}{|l|}{ Father's education, $n(\%)^{2}$} \\
\hline Up to 9 years & $122(62.9)$ & $132(75.9)$ & & $84(65.6)$ & $82(74.5)$ & \\
\hline $10-12$ years & $39(20.1)$ & $31(17.8)$ & & $24(18.8)$ & $20(18.2)$ & \\
\hline$>12$ years & $33(17.0)$ & $11(6.3)$ & 0.003 & $20(15.6)$ & $8(3.4)$ & 0.087 \\
\hline \multicolumn{7}{|l|}{ Physical Activity Index, $n(\%)^{2}$} \\
\hline Sedentary & $23(14.0)$ & $21(15.6)$ & & $5(5.9)$ & $6(7.1)$ & \\
\hline Low activity & $82(50.0)$ & $72(53.3)$ & & $40(47.1)$ & $48(56.5)$ & \\
\hline Moderately active & $49(29.9)$ & $35(25.9)$ & & $30(35.3)$ & $26(30.6)$ & \\
\hline Vigorously active & $10(6.1)$ & $7(5.2)$ & 0.398 & $10(11.8)$ & $5(5.9)$ & 0.133 \\
\hline $\mathrm{BMI}\left(\mathrm{Kg} / \mathrm{m}^{2}\right)^{1}$ & $18.1(2.7)$ & $17.7(2.8)$ & 0.062 & $18.7(2.6)$ & $18.7(2.7)$ & 0.966 \\
\hline \multicolumn{7}{|l|}{ IOTF, $n(\%)^{2}$} \\
\hline Underweight & $7(1.5)$ & $10(2.1)$ & & $2(0.7)$ & $0(0.0)$ & \\
\hline Normal & $138(29.7)$ & $157(33.8)$ & & $95(62.9)$ & $90(62.9)$ & \\
\hline Overweight & $67(14.4)$ & $41(8.8)$ & & $44(29.1)$ & $40(28.0)$ & \\
\hline Obesity & $21(4.5)$ & $23(5.0)$ & 0.054 & $10(6.6)$ & $13(9.1)$ & 0.610 \\
\hline Energy $(\mathrm{kcal} / \text { day })^{1}$ & $2091(683.9)$ & $2024.2(581.8)$ & 0.257 & $2388.0(1036.5)$ & $2475.6(684.9)$ & 0.049 \\
\hline LER, $n(\%)^{2}$ & $8(3.4)$ & $8(3.4)$ & 0.986 & $3(2.0)$ & $2(1.4)$ & 0.703 \\
\hline Total fruit $(\mathrm{g})^{1}$ & $227.9(195.1)$ & $255.6(218.2)$ & 0.161 & $221.2(268.8)$ & $188.5(218.2)$ & 0.095 \\
\hline Whole fruit $(\mathrm{g})^{1}$ & $207.4(179.2)$ & $220.6(187.8)$ & 0.444 & $175.6(206.9)$ & $162.9(193.4)$ & 0.428 \\
\hline Fruit juice $(\mathrm{g})^{1}$ & $19.8(72.2)$ & $34.8(100.5)$ & 0.070 & $45.5(162.4)$ & $25.6(83.4)$ & 0.052 \\
\hline Vegetable soup $(\mathrm{g})^{1}$ & $273.2(205.3)$ & $271.8(234.4)$ & 0.945 & $216.2(243.1)$ & $234.3(225.9)$ & 0.341 \\
\hline Total vegetables $(\mathrm{g})^{1}$ & $34.6(45.0)$ & $32.7(62.5)$ & 0.705 & $52.5(100.3)$ & $42.8(64.5)$ & 0.147 \\
\hline Green leafy vegetables $(\mathrm{g})^{1}$ & $14.0(25.1)$ & $16.2(38.0)$ & 0.465 & $52.5(100.3)$ & $42.8(64.5)$ & 0.025 \\
\hline Other vegetable $(\mathrm{g})^{1}$ & $20.6(36.5)$ & $16.7(40.6)$ & 0.284 & $26.8(66.5)$ & $25.6(43.5)$ & 0.797 \\
\hline Fruit and vegetables $(\mathrm{g})^{1}$ & $263.2(204.8)$ & $283.5(229.4)$ & 0.334 & $273.6(303.0)$ & $231.3(248.4)$ & 0.056 \\
\hline
\end{tabular}

${ }^{1}$ Results expressed as mean (sd), $t$-test or Mann Whitney test.

${ }^{2}$ Results expressed as $n(\%), \chi^{2}$ test.

vegetable (200.5 (48.3) versus $-160.8(67.6), P=0.004)$ (data not shown).

\section{Discussion}

Our study showed that a nutrition program delivered and taught by in-service nutrition-trained teachers is effective in the increase of vegetable and fruit consumption among school children.

Intervened children had a significantly higher increase in total vegetable $(P=0.018)$ and green leafy vegetable $(P=0.002)$ consumption as well as in total fruit $(P=$ $0.009)$ and fruit and vegetable $(P=0.001)$. The results regarding vegetable intake are particularly important because they are likely to be the most challenging food group to change in children [15]. If replicated, the type of intervention carried out in the present study, teaching children to increase intake of healthy high-nutrient dense foods, such as fruit and vegetable, could represent an alternative approach to primary prevention of childhood obesity [38], increase the intake of nutritionally dense foods while simultaneously decreasing intake of low-nutrient dense foods.

The effect sizes reported here ranged from small to medium and are similar to other intervention studies $[1,39$, 40]. The small effect sizes may result from an inadequate amount of the program in relation to other environmental forces, such as an environment already saturated with information from sophisticated advertisements and product 
TABLE 2: Differences between intervention and control schools on fruit and vegetables consumption at 6-month postintervention program.

\begin{tabular}{|c|c|c|c|c|c|}
\hline & Control mean $(\mathrm{SE})^{*}$ & Intervention mean $(\mathrm{SE})^{*}$ & Adjusted difference $(95 \% \mathrm{CI})^{* *}$ & $P$ & Cohen's $d$ \\
\hline Total fruit (g) & $-70.0(27.7)$ & $76.8(27.6)$ & $-282.8(-495.1 ;-70.6)$ & 0.009 & 0.47 \\
\hline Whole fruit (g) & $-59.3(24.6)$ & $26.1(21.2)$ & $-152.8(-322.7 ; 17.2)$ & 0.078 & 0.34 \\
\hline Fruit juice (g) & $-10.7(13.0)$ & $50.6(18.3)$ & $-118.8(-240.0 ; 2.4)$ & 0.055 & 0.27 \\
\hline Vegetable soup (g) & $21.4(26.2)$ & $-24.2(23.6)$ & $67.8(-109.3 ; 244.9)$ & 0.453 & 0.09 \\
\hline Total vegetable (g) & $26.7(8.8)$ & $48.3(10.3)$ & $-89.7(-164.2 ;-15.2)$ & 0.018 & 0.29 \\
\hline Green leafy vegetable (g) & $9.1(5.7)$ & $24.2(4.9)$ & $-61.3(-101.0 ;-21.6)$ & 0.002 & 0.38 \\
\hline Other vegetable (g) & $17.6(5.3)$ & $24.1(8.1)$ & $-28.4(-82.0 ; 25.2)$ & 0.299 & 0.12 \\
\hline Fruit and vegetable (g) & $-43.2(29.4)$ & $125.0(28.7)$ & $-377.5(-598.1 ; 156.9)$ & 0.001 & 0.56 \\
\hline
\end{tabular}

Notes: in the generalized linear models, children were nested within school, and an adjustment was made for children's gender, age, baseline total energy intake, parents' education, baseline BMI, and baseline measures of the dependent variable.

* Unadjusted mean.

** Differences between the control and intervention groups were adjusted for children's gender, age, baseline total energy intake, parents' education, baseline BMI, and baseline measures of the dependent variable.

promotions [41]. However, these results are important because there are countless diseases resulting from poor eating habits, including obesity $[40,42]$.

Our study has both strengths and limitations. One of the weaknesses is that we did not explore differences between schools selected and not selected to the study because of human and materials constraints. However, schools were from the same geographical area and, to the best of our knowledge, no data is available reporting significant sociodemographic and income differences between schools from this particular area. In addition, to the best of our knowledge there were no other "antiobesity" programs going on in intervention and control schools. Another weakness was the use of one weekday of dietary recall. Although repeated $24 \mathrm{~h}$ dietary recalls are required to assess the adequacy of the diet of an individual, a single record is considered suitable to estimate the consumption of food groups, and mean nutrient intake in a population, when conducted in a random sample and carefully analysed [43]. The chosen method has been widely used with a high response rate, allows for accurate diet quantification, and does not alter habitual intake $[31,32]$. Although the intervention program was based on health promotion model and social cognitive theory we did not measure the constructs of the models and demonstrate changes in those constructs. Another limitation is the possibility that our sample size was not enough to detect other significant differences than those reported. Furthermore, we failed to obtain identically equivalent groups after randomization, namely, in parents education level, mainly because we randomize by school and not by subjects, in order to avoid cross-contamination between intervention and control schools. Nevertheless, these differences were taken in account in all of the statistical models. The prevalence of low energy reporting found here was similar to that identified in earlier studies [44] and all multivariate analyses were performed both for the total sample as only for normal energy reporters.

On the other hand, the present study has important strengths that should be acknowledged. First, this study included the nutrition education program in the progression of teaching career. This probably allowed teachers to feel motivated to be part of the program. It would be desirable that other similar programs could be recognized on the career progression of teachers in Portugal and other countries in order to engage and reward teachers for their efforts. Second, in-service training to teachers was prolonged in time, and the subsequent network developed between themselves, researchers, and children was fruitful. We knew, from previous data in Portugal, that apprentice teachers did not have health promotion on their academic curricula, neither was it considered important increase to its health content [45]. Being aware of this need and that long-term interventions are more effective than those of short duration, [16] we promoted a six-month duration training program expecting teachers to become also nutrition educators. We believe this period allowed teachers to recognize just how important healthy eating habits and physical activity are. Third, our approach was to standardize recommendations to teachers, allowing them enough flexibility to create interactive interventions and pedagogic instruments to be used with children. This is contrary to previous school-based programs that have used tight controls to ensure uniform implementation but required frequent staff training and ongoing supports [46]. Other studies have confirmed the effectiveness of our model program $[18,19,22]$. Hence, this program respected school culture and was individualized to its real needs. It would be desirable that children from the present study could be followed to see whether the results still hold through life cycle, but no resources exist to further support this followup.

The World Health Organization (WHO) population goal for consumption of fruit and vegetables is $400 \mathrm{~g}$ per day [6]. There are no guidelines for how to interpret the population goals for this specific age group. Possibly, children should end up somewhere in the lower end of the range compared with the range of intake for the whole population. Due to this problem we did not judge in this study if the children achieved the WHO population goal. Previous studies stated 
that vegetable intake was clearly lower than fruit intake [10]. Likewise, in this study a large proportion of children had a higher intake of fruit compared to vegetables.

We observed an interaction of weight status on the outcome measures. Intervened obese (overweight and obese) children reported a significantly higher consumption of total vegetable, green leafy vegetable, total fruit as well as fruit and vegetable than normal weight children. This result is relevant because evidence suggests that consumption of lowenergy and nutrient-rich foods such as fruit and vegetable is recommended as an obesity prevention approach [6].

It has been argued that teachers do not have enough time and energy to provide interventions as dedicated interventionists [16, 17]. However, this study contributes to clarify the scant evidence of the effect of nutrition education-only programs delivered by teachers [15] and provides support for their role in improving children eating habits. Moreover, the previous educational sessions with the researchers provided the information needed to be properly applied in the school's curriculum. Teachers, in turn, were able to adapt the topics according to children's needs throughout the year and not just in a single moment or a specific session.

\section{Conclusions}

Our program targeting classroom teachers as dedicated interventionists had significant effects on children's fruit and vegetables consumption. Although this program cannot be used to demonstrate life-long changes in eating habits, it does show the ability of an education program to impact on diet at a crucial life stage when eating habits are being formed. Future studies should involve a follow-up period to see if the significant effect can be sustained for a longer term. Also, the lack effect of the program suggests that a more intense or longer program may be needed to reinforce this trend. Another important direction could be to centre the program on other aspects like school environment, physical education classes or on the environment beyond schools, such as homes and stores. Finally this program offers promise for use in youth obesity prevention and highlights the need for continued emphasis on nutrition education and health promotion at school.

\section{Acknowledgments}

The authors express their deepest appreciation to all the collaborators who assisted the development and implementation of the study, namely, Célia Oliveira, Fernando Ferreira, and Elisete Ferreira. Special thanks are extended to Dra. Paula Neves and Dr. Benjamim Sampaio for the assistance provided throughout this program. The authors are grateful acknowledgement goes to all the children and their parents for the participation in this study, school administrators, and school staff for the opportunity of the implementation of the study.

\section{References}

[1] D. A. Gentile, G. Welk, J. C. Eisenmann et al., "Evaluation of a multiple ecological level child obesity prevention program: switch what you do, view, and chew," BMC Medicine, vol. 7, article no. 1741, p. 49, 2009.

[2] Y. M. Tantamango, S. F. Knutsen, W. L. Beeson, G. Fraser, and J. Sabate, "Foods and food groups associated with the incidence of colorectal polyps: the adventist health study," Nutrition and Cancer, vol. 63, no. 4, pp. 565-572, 2011.

[3] R. Barros, A. Moreira, J. Fonseca et al., "Adherence to the Mediterranean diet and fresh fruit intake are associated with improved asthma control," Allergy, vol. 63, no. 7, pp. 917-923, 2008.

[4] A. Oliveira, C. Lopes, and F. Rodríguez-Artalejo, "Adherence to the Southern European Atlantic diet and occurrence of nonfatal acute myocardial infarction," The American Journal of Clinical Nutrition, vol. 92, no. 1, pp. 211-217, 2010.

[5] L. Serra-Majem, B. Roman, and R. Estruch, "Scientific evidence of interventions using the Mediterranean Diet: a systematic review," Nutrition Reviews, vol. 64, no. 1, Supplement 1, pp. S27-S47, 2006.

[6] WHO, Diet, Nutrition and the Prevention of Chronic Diseases, WHO, Geneva, Swetzerland, 2003.

[7] J. Brug and A. Oenema, "Healthful nutrition promotion in Europe: goals, target populations, and strategies," Patient Education and Counseling, vol. 63, no. 1-2, pp. 255-257, 2006.

[8] C. Currie et al., "Inequalities in young people's health: HBSC International," Report from the 2008/2006 Survey, WHO Regional Office for Europe, Copenhagen, Denmark, 2008.

[9] F. Branca, H. Nikogosian, and T. Lobstein, The Challenge of Obesity in the WHO European Region and the Strategies for Response Summary, WHO, Copenhagen, Denmark, 2007.

[10] A. Yngve, A. Wolf, E. Poortvliet et al., "Fruit and vegetable intake in a sample of 11-year-old children in 9 European countries: the pro children cross-sectional survey," Annals of Nutrition and Metabolism, vol. 49, no. 4, pp. 236-245, 2005.

[11] L. L. Birch and J. O. Fisher, "Development of eating behaviors among children and adolescents," Pediatrics, vol. 101, no. 3, pp. 539-549, 1998.

[12] S. J. te Velde, J. W. R. Twisk, and J. Brug, “Tracking of fruit and vegetable consumption from adolescence into adulthood and its longitudinal association with overweight," British Journal of Nutrition, vol. 98, no. 2, pp. 431-438, 2007.

[13] N. Lien, L. A. Lytle, and K. I. Klepp, "Stability in consumption of fruit, vegetables, and sugary foods in a cohort from age 14 to age 21," Preventive Medicine, vol. 33, no. 3, pp. 217-226, 2001.

[14] L. A. Lytle, S. Seifert, J. Greenstein, and P. McGovern, "How do children's eating patterns and food choices change over time? Results from a cohort study," American Journal of Health Promotion, vol. 14, no. 4, pp. 222-228, 2000.

[15] E. van Cauwenberghe, L. Maes, H. Spittaels et al., "Effectiveness of school-based interventions in Europe to promote healthy nutrition in children and adolescents: Systematic review of published and grey literature," British Journal of Nutrition, vol. 103, no. 6, pp. 781-797, 2010.

[16] E. Stice, H. Shaw, and C. N. Marti, "A meta-analytic review of obesity prevention programs for children and adolescents: the skinny on interventions that work," Psychological Bulletin, vol. 132, no. 5, pp. 667-691, 2006.

[17] M. Sharma, "International school-based interventions for preventing obesity in children," Obesity Reviews, vol. 8, no. 2, pp. 155-167, 2007. 
[18] F. Angelico, M. Del Ben, L. Fabiani, P. Lentini, F. Pannozzo, and G. C. G. Urbinati Ricci, "Management of childhood obesity through a school-based programme of general health and nutrition education," Public Health, vol. 105, no. 5, pp. 393-398, 1991.

[19] A. Boaz, S. Ziebland, S. Wyke, and J. Walker, "A "five-aday" fruit and vegetable pack for primary school children. Part II: controlled evaluation in two Scottish schools," Health Education Journal, vol. 57, no. 2, pp. 105-116, 1998.

[20] D. D’Addesa, D. Martone, F. Sinesio et al., "Testing a nutritional and taste education intervention approach to increase vegetables and fruit consumption among children," Annali di Igiene, vol. 20, no. 2, pp. 159-169, 2008.

[21] R. T. Mangunkusumo, J. Brug, H. J. De Koning, J. Van Der Lei, and H. Raat, "School-based internet-tailored fruit and vegetable education combined with brief counselling increases children's awareness of intake levels," Public Health Nutrition, vol. 10, no. 3, pp. 273-279, 2007.

[22] M. F. Panunzio, A. Antoniciello, A. Pisano, and S. Dalton, "Nutrition education intervention by teachers may promote fruit and vegetable consumption in Italian students," Nutrition Research, vol. 27, no. 9, pp. 524-528, 2007.

[23] M. F. Panunzio, A. Antoniciello, E. P. Cela et al., "15-week long school-based nutritional education program to promote Italian primary schoolchildren's fruit and vegetable intake," Annali di Igiene, vol. 22, no. 5, pp. 481-484, 2010.

[24] M. C. Turnin, M. T. Tauber, O. Couvaras et al., "Evaluation of microcomputer nutritional teaching games in 1,876 children at school," Diabetes and Metabolism, vol. 27, no. 4 I, pp. 459464, 2001.

[25] L. Wells and M. Nelson, "The National School Fruit Scheme produces short-term but not longer-term increases in fruit consumption in primary school children," British Journal of Nutrition, vol. 93, no. 4, pp. 537-542, 2005.

[26] N. J. Pender, Health Promotion in Nursing Practice, Prentice Hall, NJ, USA, 3 ed edition, 1996.

[27] A. Bandura, Social Foundations of Thought and Action: a Social Cognitive Theory, Prentice Hall, NJ, USA, 1986.

[28] WHO Expert Commitee, Physical Status: the Use and Interpretation of Anthropometry, vol. 854, Who, Geneva, Swetzerland, 1995.

[29] T. J. Cole, M. C. Bellizzi, K. M. Flegal, and W. H. Dietz, "Establishing a standard definition for child overweight and obesity worldwide: international survey," British Medical Journal, vol. 320, no. 7244, pp. 1-6, 2000.

[30] H. Pan and T. Cole, "lmsGrowth, a Microsoft Excel add-in to access growth references based on the LMS method," (Version 2.68) 2009, www.healthforallchildren.co.uk/.

[31] S. Kranz and A. M. Siega-Riz, "Sociodemographic determinants of added sugar intake in preschoolers 2 to 5 years old," Journal of Pediatrics, vol. 140, no. 6, pp. 667-672, 2002.

[32] S. Gómez-Martínez, A. Martín, J. Romeo et al., "Is soft drink consumption associated with body composition? A crosssectional study in Spanish adolescents," Nutricion Hospitalaria, vol. 24, no. 1, pp. 97-102, 2009.

[33] W. N. Schofield, "Predicting basal metabolic rate, new standards and review of previous work," Human Nutrition. Clinical Nutrition, vol. 39, pp. 5-41, 1985.

[34] WHO, FAO, and UNU, "Human energy requirements," Report of a Joint FAO/WHO/UNU Expert Consultation, FAO, Rome, Italy, 2004.
[35] G. R. Goldberg, A. E. Black, S. A. Jebb et al., "Critical evaluation of energy intake data using fundamental principles of energy physiology: 1. Derivation of cut-off limits to identify under-recording," European Journal of Clinical Nutrition, vol. 45, no. 12, pp. 569-581, 1991.

[36] J. Mota and C. Esculcas, "Leisure-time physical activity behavior: structured and unstructured choices according to sex, age, and level of physical activity," International Journal of Behavioral Medicine, vol. 9, no. 2, pp. 111-121, 2002.

[37] J. Cohen, Statistical Power Analyses for the Behavioral Sciences, Lawrence Erlbaum Associates, Hillsdale, NJ, USA, 2 ed edition, 1988.

[38] L. H. Epstein, C. C. Gordy, H. A. Raynor, M. Beddome, C. K. Kilanowski, and R. Paluch, "Increasing fruit and vegetable intake and decreasing fat and sugar intake in families at risk for Childhood Obesity," Obesity Research, vol. 9, no. 3, pp. 171178, 2001.

[39] T. Baranowski, J. Mendlein, K. Resnicow, E. Frank, K. W. Cullen, and J. Baranowski, "Physical activity and nutrition in children and youth: an overview of obesity prevention," Preventive Medicine, vol. 31, no. 2, pp. S1-S10, 2000.

[40] M. Story, K. M. Kaphingst, and S. French, "The role of child care settings in obesity prevention," Future of Children, vol. 16, no. 1, pp. 109-142, 2006.

[41] G. Sorensen, K. Emmons, M. K. Hunt, and D. Johnston, "Implications of the results of community intervention trials," Annual Review of Public Health, vol. 19, pp. 379-416, 1998.

[42] J. C. Eisenmann, "Insight into the causes of the recent secular trend in pediatric obesity: common sense does not always prevail for complex, multi-factorial phenotypes," Preventive Medicine, vol. 42, no. 5, pp. 329-335, 2006.

[43] R. S. McPherson, D. M. Hoelscher, M. Alexander, K. S. Scanlon, and M. K. Serdula, "Dietary assessment methods among school-aged children: validity and reliability," Preventive Medicine, vol. 31, no. 2, pp. S11-S33, 2000.

[44] M. D. Kontogianni, N. Vidra, A. E. Farmaki et al., "Adherence rates to the mediterranean diet are low in a representative sample of greek children and adolescents," Journal of Nutrition, vol. 138, no. 10, pp. 1951-1956, 2008.

[45] V. Rodrigues et al., "Os professores e a educação/promoção para a saúde," in Novas Realidades, Novas Práticas. Actas do seminário Internacional de Educação Física, Lazer e Saúde, B. Pereira, G. S. Carvalho, and V. Pereira, Eds., Instituto de Estudos da Criança da Universidade do Minho, Braga, Portugal, 2007.

[46] D. M. Hoelscher, H. A. Feldman, C. C. Johnson et al., "Schoolbased health education programs can be maintained over time: results from the CATCH Institutionalization study," Preventive Medicine, vol. 38, no. 5, pp. 594-606, 2004. 


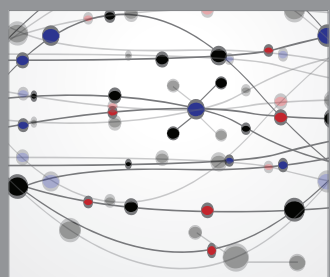

The Scientific World Journal
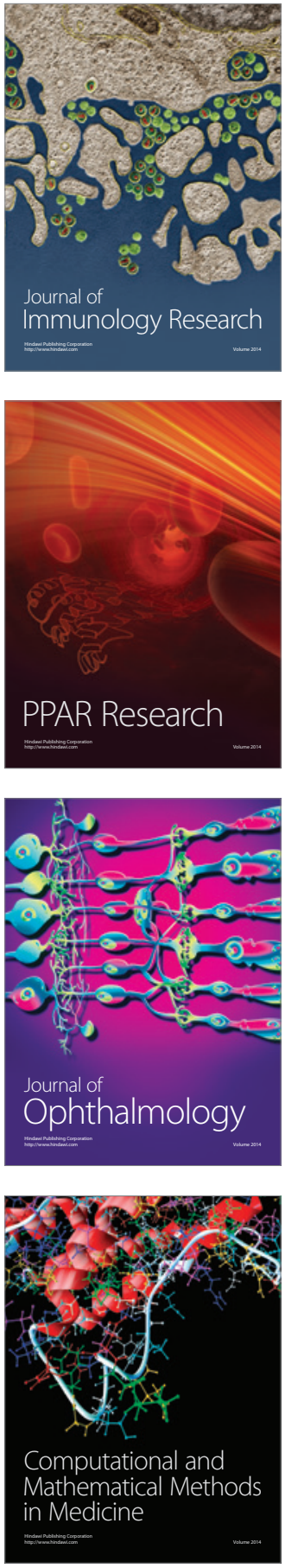

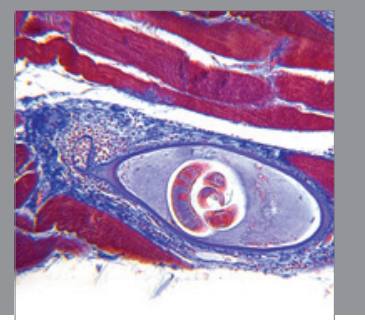

Gastroenterology

Research and Practice
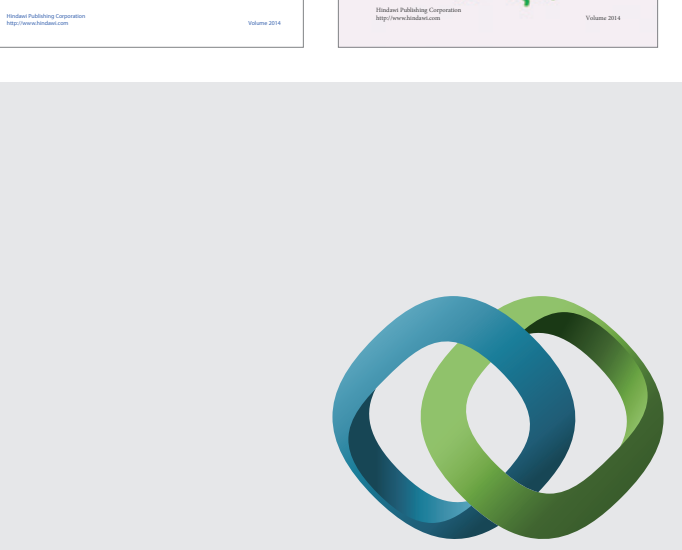

\section{Hindawi}

Submit your manuscripts at

http://www.hindawi.com
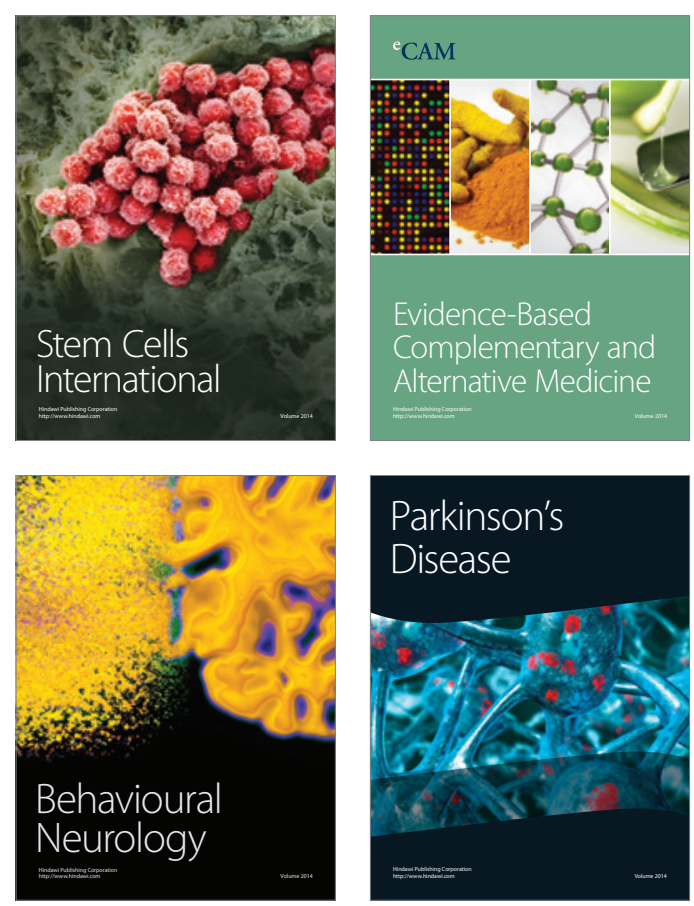

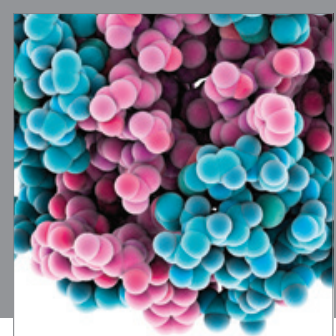

Journal of
Diabetes Research

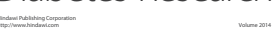

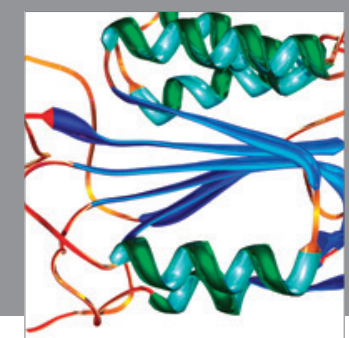

Disease Markers
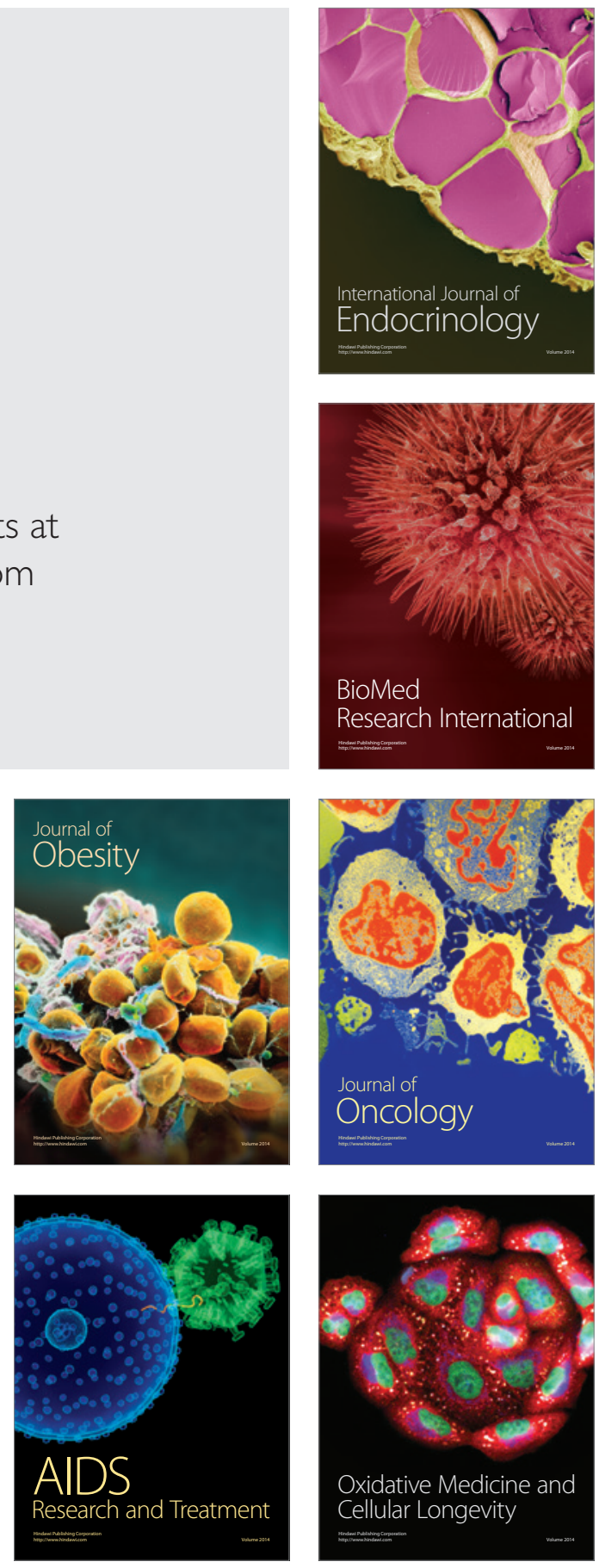